\title{
Algunas consideraciones sobre el desprendimiento prematuro de la placenta normalmente insertada
}

\author{
PoI RAMON FRANCISCO SANCHEZ
}

Jefe de Clmica Obstetrica de la Universidat Nacional

El ciccidente obstetrico que sirve de tema a esta breve monogratici, au.: no tiene una denominación aceptadc unánimemente por todos los tratadistas, y en relación con su frecuencic también las cifras varian de acuerac con el criterio que se tenga sobre $\epsilon$. particular. Hematoma retroplacentorio baútizólo Couvelaire; hemorragic y apoplejia útero-placentarias designólo Turene: algunos hablan de hemoragia retroplacentaria, otros de desprendimiento de la placenta normaimente insertada. De Lee lo califica de abruptio placentae, Recasens lo llama desprendimiento preternatural de la placenta $y$, en fin, Stabile al rele-rirse a él lo titula despréndimiento prematuro o precoz de la placenta inse:Ta en el cuerpo del útero, según que el tenómeno aparezca durante el errbarazo o en el curso del parto (1).

Siguiendo a la mayoría de los expositores adopto el título que encabe. 20 este trabaio, que se refiere a caeos de desprendimiento observados en $\mathrm{e}$ ! Instituto de Protección Materna e iniantil de Bogotá, donde, en calidad de Jete de Clínica Obstétrica, tuve opo: tunidad de tratar algunos muy de corca.
En el mencionado Instituto la trecuencia, incluyendo los casos leves, sin ningún aparato sintomático y sin el dramatismo que rodea los graves, es bastante escasa. Corral Maldonado (2) encontró un dos por mil en 1947-48 (Servicio del Protesor Rodriguez Aponte), y en 1951 y lo transcurrido de 1952 (cinco meses y medio) sobre 10.865 pacientes entradas a todo el Instituto, Ja proporción tue de un poco márs de uno por mil aproximada a la que apunta Botella Llusia on su aPctología Obstétrica (3).

La trecuencia señalada por Stabile es de uno por cinco mil; Holmes trae un dos por ciento: Frank! un nueve por mil; Ginglinger trece por mil: Pankow cuatro por mil y Albeck sele por mil. indices mayores que los de nuestro medio, quizás porque en otras lattudes se acude con más fervor al examen anatomopatológtco.

Nubiola en su estudio sobre la apoplejía úteroplacentaria, incluído en su ya fomoso *Tratado de Obetetriclav (4). hace una breve historia del síndrome. Desde 1697 el legendario Mauriceon. con tla sagacidad clínicar de los vielos expositores de la encuela tranceso. vislumbró la existencia del despren, 
estableció la diferencia esencial entro la hemorragia por desprendimiento $\mathrm{y}$ la debida a la placenta previa, y llamola accidental. Winter en 1885 sugirio las relagiones que pudierá tener con la uremia. Couvelaire en 1911 la wizo tema de un insuperable análisis y describir el litero apoplético y a int su nombre a las formas graves, n la anatomía patológica demostró yue el hematoma retropiacentario se compañaba de una hemorragia difusc en pleno músculo uterino. Posteriormente Kower, Essen-Moller Y Zweifel describieron desgarros de la serosa uterina. En uno de los casos que presento, la infiltración sanguínea llegaba hasta la serosa parietal y a traves de una hernia umbilical se veíx que la sangre estaba ya en la gran ctvidad peritoneal (observación número cinco).

Etiologia. La etiología del desprendimiento prematuro de la placenta normalmente insertada ha sido incriminada a taiferentes factores, desde las causas mecánicas o externas y los tgumatismos, incluídos los traumatisnos obstétricos, v. gr. las maniobras ara una versión, hasta las toxemias iravidicas y el sindrome cardio-renal, a más de afecciones uterinas $y$ del huevo.

Sintetizando, podría decirsé que las más acusadas serian las mecánicas. como maniobras bruscas conducentes a una versión, la depleción uterina por la bruscer evacuación de un hidramnios o por la salida de un primèr feto gemelar, la acción de atirantamiento por un cordón corto, los traumatismos (golpes, caídas, etc.) el orit gen local por endometritis y lesiones las de linaje tóxico, que han hecno distinguir al desprendimiento prematuro con el nombre de eclampsia hemorrágica. En la mayoría de las pacientes que sirven de demostración de este trabajo se observaron edemas, pero ninguna fue protagonista de la sintomatología descrita como eclampsismo con todo ese cortejo heráldico de las convulsiones (ver nota al final de este estudio).

Sintomatologia. El cuadro clínico tiene manifestaciones diversas, désde una escena envuelta en discreta ponumbra asintomática, apenas diagnosticable a posteriori, cuando se examina la placenta, hasta presentarse como dramático conflicto de tánta rapidez e intensidad que la madre muere cunque se acuda a prestarle auxilios inmediatos.

Como se observan casi siempre las formas aparentes, llama la atención la anamnesis: aparición de un súbito dolor con sensación de angustia, luégo la permanencia y constancia del dolor generalizado a todo el abdomen. Al examen directo se nota que el útero está duro, leñoso, con tono basal muy elevado y que su palpación exacerba el dolor. Hay también metrorragia, escasa casi siempre, pero no en todas las veces es sintoma del comienzo sino terminal. El pulso en circunstancias no desesperadas puede permanecer stn modificaciones notables y la tensión arterial por lo generáles variable. En la mayoria de las ocasiones se ven edemas y el hallazgo de albúmina no es constante. La auscultación fetal es negativa cuando hd transcurrido cierto tiempo desdo la iniciación del accidente, y si éste no ha aparecido simultáneamente con el 
En las pacientes observadas un sintoma que no faltó fue la hipertonia uterina. En E. Q. (observación número cinco), no se la vió a su llegada al Servicio, pero después se hizo aparente hasta tal punto que al principio ta paipacion permitía diagnosticar una presentacion de vértice y dos horas despues la dureza de la matriz habia llegado al extremo que era imposible saber a ciencia cierta qué polo fetal se hallaba en relación con el estrecho superior. En esa misma enferma también fue notable el fenómeno de que la hemarragia genital se presentó tardia; principió 10 horas después de iniciada la complicación del desprendimiento, no abstante que habia sido completo y que la matriz se encontraba en su totalidad infiltrada.

Cuando el accidente comentado es grave, se acompaña de síntomas del shock: pulso filiforme. tensión inmensurabie; palidez de cera, indecible sensación de angustia, a veces indiierencia asiática ante la inminent desintegración anímica, otras una agitación irrétrenable, expresión quizás de la agonía, de la lucha postrera frente a la anoxia que azota los tejidos.

Un signo muy notable que estuvo presente en los casos por mí observados fue-el paulatino y continuo crecimiento de la matriz, armónico con la hipertonía-ascendente, y que -confirmó más de una presunción diagnósticca.

Ia evolución del sindrome puede suspenderse con total restablecimiento de ta enterma $y$ con final de parto espontáneo aun con feto vivo, cuando to un casc en que el parto fue espo: táneo con feto muerto y otro en $\theta$ ! que además habia una procidencia del cordón y que terminó, ante la so:presa del interno,-con feto vivol En al resto del historial se ve cómo casi siempre se intervino, ya con fórceps, or con cesarea sola o seguida de histerestomia.

Otras veces la evolución sigue un curso lento y la situación va perdiendo la gravedad hasta hacerse favoráble o se estaciona sin adquirir reheves inquietantes. El colega Hernando Gorrea, en la Clínica Primero-de Mayo, tuvo oportunidad de atender a una paciente, C. M. S., con carnet 73.085, cuya historia reza: 8 de $\mathrm{ma}$ yo de 1952. Ayer tarde sutrió una caida. Desde hoy a las 5 a. m. ha estado con hemorragia. Dolor continuo en la región lumbar. No se oyen ruidos fetales. Altura uterina de 32 centímetros. Tensión arterial de 125 por 85. Pulso, 72. Septigestante. Amenorrea desde el 31 de agosto de 1951. Entrada a las 10 a. m. A las 11 y 30 el útero estaba tétanizado $y$ el cuello grueso con un centimetro de dilatación $y$ membra. nas integras. Se prescriben dos ampolletas de edhanol y una ampolleta de mortina de un centigramo. A las $12 \mathrm{y}$ 30 . contracciones fuertes cada dos minutos y con 45 segundos de duración y cuello con 4 centímetros. Hemorragia genital. A las 14 y 15 se efectuó espontáneamente la expulatón de un feto muerto de 2.600 gramos y 47 centímetros de talla y la Inmediata sailda de la placenta. El 13 de mayo la paciente salió de la clínica. El puerperto fue completamente normal. El prote- 
principios del presente ano. Ramirez Merchón en su trabajo de agregación (9) se detiene un poco en el análisis del desprendimiento placentario y comenta dos historias que vienen a coroborar lo proteiforme de la sintomatología y el eclecticismo en la conducta.

Finalmente, la evolución puede ser rápida, con inmediata muerte del feto. y con excesiva gravedad de la madre. lo que obliga a no guardar una actinud de espectativa sino a intervenir cuanto antes. Es lo que sucedió con casi todas las enfermas vistas en el Instituto de Protección Materna e Infantil.

Patogenia. Dexeus Font (5) dice que el estudio de la patogenia del desprendimiento prematuro de la placenta normalmente insertada tropieza con el mismo valladar de las gestosis. Habla de una supuesta hiperactividad corial y de una sensibilización a hipotéticos agentes tóxicos a la vez que invoca la opinión de Couvelaire al comentar que el desprendimiento prematuro de la placenta no es un proceso local síno manifestación de un estado tóxico general. Por otra parte considera que la síflis actúa como coadyuvante del proceso tóxico para lesionar los vasos y que en la multi. gestación hay además factores vasculares y endometríticos que predisponen a la aparición del hematoma retroplacentarto.

Botella, en su obra citada, considera que precisamente una manifestación de gestosis es la tragilidad capilar $\theta$ Individualiza la diatesis hemorrági. ca grovidica. El mecanismo y el ager. tan la accion a la hipentusiaminem:

Anatomia patológica. Según la definición, el sindrome consistiria en unc desinserción central o lateral de ?a placenta, consecutiva o precedente a la hemorragia, lo que dará por consecuencia una pérdida total o parcial de las relaciones placento-maternus con la muerte tetal. Pero no se tratc: en verdad de un desprendimiento cuando ta etiología es loxémica, sine de una real destrucción de ta placenta y de una sustitución de tejido par el hematoma. No hay una simple apos:ción de un coágulo sino una verdadera histolisis que se evidencia cuando se desprende aquél: se ve entonces una excavación que estaba llenada por la sangre coagulada. El examen microscópico expone una irrupción de sangre fetal en los lagos y estroma maternos, estasis venosa en las vellcsidades y a veces en la periteria de la región alecta una pseudocápsula corrpuesta de vellosidades comprimidas y una degeneración hialina y oranulosa. Al examen macroscópico siempre se manifiesta un códouto de dimensiones variables, lo que permite hacer el diagnóstico retrospectivo cuando la sintomatología fue tan leve que no se mostró claramente. En las otras circunstancias es un factor confirmativo del diagnóstico jünto con e! simultáneo alumbramiento o con el hallargo de la placenta desprendida.

No sólo la placenta sufre modificaclones on el curso evolutiva del sindrome estudiado. Las lesiones hemoirtaicas infiltrantes pueden extenderse a Ia matrir total a parcialmente, a los lifamentos anchos, a las víceras abdominales y aun formarse un he 
ces citada, el signo de Hotstatter se encuentra. En esa paciente la patogenia del signo debiase a la delgadez de la pared por la hernia umbilical. En esos casos de infiltración uterina es cuando se habla de la apoplejía útero placentaria, exigente las más de las veces de una amputación de la matriz.

Pronóatico. Entre nosotros la muerte fetal ocurrio en el $80 \%$ de los casos y la materna en dos casos sobre 12 $(16 \%)$. La estadística de Corral Maldonado (2) en lo que atañe al feto, fue más alta, pero él en los siete casos que observó no fuvo ninguna defunción materna.

Las estadísticas extranjeras anotan lo siguiente:

\section{Mortalidad materna:}

Winter, $50 \%$.

Pankow, $3.1 \%$.

Salomons, 3.7\% .

Brindecu, $4.6 \%$,

De Snoo, 6\%.

Le-Lorier, $6.2 \%$.

Liever, $11 \%$ (6).

Tabares, $1.6 \%$

Paracchi, $7.5 \%$.

Mariño Donato, $10.2 \%$.

Dexeus Font, $8.6 \%$ (5)

\section{Martalidad folal}

Lteven, $56 \%$.

Franizi, 62\%.

Brindecu, $72 \%$.

Ponkow, $76 \%$.

Paracch, 70\%.

Martho, 67.6\%.

Dexeus, $84 \%$.

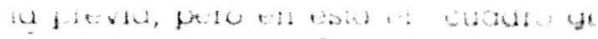

neral de la paciente es armónico con la hemorragia, es decir, los sintomas alarmantes en cuanto a la mujer $\mathrm{son}$ proporcionales a la cantidad de la pérdida sanguinea; además la ruptúra de las membranas en la placenta previa generalmente aminora los sintomas y el útero en la placenta inferor no se tetaniza. En una de las historias que comento hubo vacilacior en el diagnóstico antes de la ruptura de las membranas, pero la intensificación de la hemorragia, ya con bolsa rota, simultáneamente con las cantracciones inclínó el pensamiento hacic un desprendimiento parcial labservación número 4).

La ruptura uterina puede también presentarse a confusión, como lo demuestra el relato del Profesor Hernar do Acosta en sus clases de obstetricia en 1941 (7), en el que una ruptura espontánea de la matriz duranie el embarczo hizo intervenir por vía alta por haberse diagnosticado desprendimiento prematuro de la placenta. En la ruptura también es aparente ol cua: dro de abdomen agudo y hay hemorragió genital escasa. los ruidos cardiacos tetales desaparecen y hay inlenso dolor. Pero éste generalmente es pasajero y se puede tocar al feto. en canto que en el desprendimiento prematuro de la placenta el dolor es constante y cuando la hipertonía ha instalado la palpactón del feto es dikell o imporible.

DI hidramnios agudo asosiado a amencea de parto, prematuro o no, puede contundirse con el sindrome del desprendimiento placentario, - 


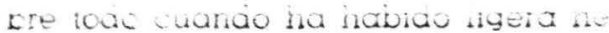

morragia genital. A la clínica Primero de Maro tue una paciente con altura uterina de 40 centímetros, con hipertonía uteina, auscultación fetal negativa y con anteredentes de pérdidas hematicas $y$ de haber cesado súbitamente los movimientos tetales mas con tension arterial no muy baja y 150 pulsaciones por minuto. Trasladose al Hostutal de San Juan de Dios y cill tue intervenida por la impresión clínica de desprendimiento de "placenta: se hallo un hidramnios acuudo con teto muerto.

Tratamiento. No hay acuerdo sobre la conducta frente al desprendimiento piematuro de la placenta normalmente insertada. Algunos son ultraconservadores y consideran la cesárea-como exceso de terapéutica, $y$ se inclinan hacia el uso de antiespasmódicos y de la rotura precoz de las membranas en el comienzo del trabajo, y hacia el fórceps o la versión cuanda la dilatación es compleia o fácilmente completable. Empero, en los casos muy graves esos mismos conservadores a outrance no pueden menos de aceptar la cirugia, que los intervencionistas extremos la llevan hasta la histerectomía sistemát:ça.

Dexeus Font recomienda tob. cit.), iniciar siempre el tratamiento médico que solo chandona cuando el cuadro se agrava, y que resume asi:

"Reposo absoluto en cama, inyección de morlina $\theta$ ingestión de bebidas cizucaradas.

Inyección intravenosa de 200 c. c. de suero glucosado at $15 \%$, al que (8) asociara un inyectable de veritol, 6 simpaich $L$

veces al día.

Si se iniciá el parto, administración de antiespasmódicos -(espasmalgina. dolantina, glicerofostato-sódico).

Caso de que la dilatación no pragrese, rotura artificial de la bolsa.

Dejar que el alumbramiento se haga espontáneamente. $Y$ und vez to:minado inyección de ergometrina

Si el útero se contrae mal, taponamiento útero-váginal epretado, con valvas y anestesia, llevado a cabo sin demasiada tardanza. Una faja abdominal completará los efectos del taponamiento: Preparación para la laparotomía. Si la hemoriagia persiste a pesar de todo, histerectomía precedida o acompañada de una transfusión.

Manuel Luis Pérez diferencia el desprendimiento con síntomas leves de! desprendimiento con sintomas graves, $y$ si bien en-los casos muy tenues optá por la expectación, en los que se presentan con sintomatología clara sí es francamente intervencionista, de- cide la evacuación rápida del útero. sea con cesárea vaginal o abdominal, o con el parto metódicamente rápido. según las circunstancias.

Botella Llusiá es más categórico y atirma que ela via abdominal es el procedimiento de elección utilizando la cesárea sola o seguida de histerecto. iaía segúr gise ol utero se contraiga normalmente o no.

En los casos directamente atendidos seguí la líned de conducta francamente intervencionista de acuerdo con el Protesor Carlós Jullo Moilica: dos pacientes fueron histerectomizadas.'por el Profesor y a dos hice cesárea sim- 
Gunque habia zonas equimóticas. Estas hicieron un post-operatorio normal; de aquéllas una murió después de operada, mas es preciso dejar constancia de que no se le hizo transusion por no haber sangre en el hos:

H. sonalmente soy partidato de la : :ndr evacuación ute-ina con cesátec s! el accidente surgio en el embarazo o si la insuliciente dilatación 0 Ia taita de encajamiento no indican la apolicación del tórceps. Hago abstracción del feto, pues al tratamiento ante todo debe dirigirse a buscar la salvación de la madre. No importa que el feto haya muerto para hacer una cesárea segmentaria, pues como en la placenta previa central total, in teresa la vida de la mujer y por tanto es lógico acudir al sistema menos peligroso y que dé más garantias. Peli gro inmediato en el desprendimiento prematuro placentario es la infiltración sanguínea de la musculatura uterina con la alteración histológica consiguiente. ¿Cómo saber que no hay una apoplejía uterina o que la matriz no va a sufrir cambios notables en su constitución en el lapso de la paciente $\gamma$ prolongada espera? Interviniends por via abdominal se verá directamente el estado del útero y sí vale la pena conservarlo o no. Claro es que encciada la presentación o estando adelantada la dilatación cervical en grado mayor que permita la ayuda del tórcepes se coeptond́-este concurso, pero vigilando estrechanente a la paciente durante varias horas después del parto a fin de actuar interviniendo por vía alta si la hemorragia se hicie- ducta intervencionista no me adhiero a los particarios de la histerectomia sistemática. No veo la razón de amputar una matriz que se retrae blen. signo de que sus alteraciones no son irremediables, además de que la histerectomia es asaz traumatizante $y$ pued cgravar el estado precario de la naciente prolongando el acto quiruraco $\sin$ perentoric necesidad.

Condicion casi indispensable para el buen exito es la transfusion apenas se haga el diagnóstico y en el curso de la intervención quirúrgica. Cuando se procedió asi los post-operatorios fueron periectos y fas enfermas se repusieron prontamente de su tremenda inferioridad vital. Durante los días siguientes a la operación hay que hidratarlas y suministrarles antibióticos.

Desde ningún punto de vista me parece indicada la cesárea vaginal, que si permite la rápida evacuación del útero no visualiza las lesiones de este órgano y por ende la incertidumbre sigue en pie. Tampoco es mótodo incontrovertible la versión si recuerdan la hipertonicidad uterina y la alteración histológica del miometrio. No es cuerdo para huír de un peligro arrojarse en uno mayor. Por eso cemparto la opinión de Corral Maldonado cuando dice: Por el hecho de que en algunos casos de desprendimiento prematuro de la placenta se haya podido recurrir a la versión como conduc. ta y be haya obtenido exdto matorno $y$ fetal, no se puede preconirar la versión en esta terrible complicación, ya que muy fácilmente puede productrse la ruptura uterina con todas sus graves consecuenclas, (8). 


\section{CASOS CLUNICOS}

\section{Obeervación número 1}

Servicio del Profesor Mojica. Marzo 16/51.

B. L. de P. Decigestante de 40 años. Menarquia a los 12 años. Ciclo 20/8. $\mathrm{Sin}$ dato de amenorrea. Sin edemas. 120 pulsaciones por minuto. Altura uterina de 35 centimetros. Utero blando, globuloso, de superficie regular. Presentación celálica. Auscultacion fetal positiva. Llega al Servició sangrando abundantemente y sin dolores uterinos. La matriz es palpable y sa aprecia una presentación de vértice izquierda móvil. Ante la hemorragia indolora y persistente se piensa en una placenta previa, en ruptura de várices varginales $y$ en posible desprendimiento de la placenta. Los ruidos cardíacos fetales aunque un poco lentos son perceptibles. Al tacto vaginal: cuello grueso, semi-borrado, con dos a tres centímetros de dilatación v membranas íntegras. Hay una solución de continuidad en la parte dere: cha del reborde posterior, pero no se tocom cotiledories placentarios. Al espéculum se nota el desgarro cervical con bordes traumatizados y sangrantes y que también sale sangre de la cavicad uterina. De un momento a otro, como a la media hora de la llegada al Servicio, la enferma comienza $a$ guenarse de dolores lumbares y $a$ empclidecer, a la vez que la matriz va subiencio de tano basal. Aunque ol corczón felal se negativiza se hace una laparotomía previo dictamen de desprendimiento prematuro de la placenta y se encuentra que la matriz está apoplética. Se hace una cesárea corporal y extrae un teto muerto de ción diagnóstica. Como la matriz ne se contrae y está infiltrada totalmente, se resuelve practicar una histerectcmía sub-total. Siete horas después la enterma muere.

La historia precedente sirve para demostrar que no siempre desde el principio de la complicacion se siente intenso dolor abdominal y que tampoco es constante la hipertonia se manihes te rápidamente. $Y$ se trataba de ur desprendimiento grave, tánto que yc habia apoplejía uterina con bastantes horas de duractón, pues no es po sible concobir que en el curso de tres horas, transcurridas entre la llegada al servicio y la intervención, la inilitración sanguinea hubiera alcanzado tamañas proporciones. Cuánta razon tiene el Prolesor Botella al decir en si: tratado de Patología Obstétrica (3): -Actualmente hay una gran tendencia a tratar la placenta previa par medio de la cesárea abdominal. Esta ter. dencia, aparte de otras razones, thene en su apoyo el hecho de que antiguamente muchos casos de apoplejía úteroplacentaria se diagnosticaban como placenta previa; se les trataba por víc. baja, y luégo sobrevenian estas hemorragias atónicas del alumbramiento. En aquel entonces se supuso que se trataba de una tendencia especial de las placentas previas a producir hemorragias atónicas, pero la realidad es que se estaba, sin saberlo, ante casos de abiuptio placentae, Al generalizarse el empleo de la cesćrea, ha descublerto que en un 15 a $20 \%$ do los casos diagnosticados de placentia previa, la inserción placentaria era normal, existiendo únicamente un desprendimiento prematuro de una placenta normalmente insertada. La ce- 
observa: el útero y hacer el diagnóstico dilerencial exacto, verificando la histerectomia, si el estado de aquél lo aconsejas.

La pacienté murio por talta de transtusion. Casos más graves tratados con ut precioso recurso tuvieron exito rotundo desde el punto de vista materno.

\section{Observación número 2}

\section{Servicio del Profesor Rodriguez. Marzo $12 / 52$.}

A S.L. de 39 años. Décimo embara zo. Ultima menstruación ef 3 de julio de 1951. Con edemas. Altura uterina de 34 centímetros. Matriz de consistencia dura. Presentación cefálica alta. Auscultación fetal negativa. Tensión arterial de 14 por 11. Pelvimetria de 23-27-3i-20. La enferma relata que despues de sus ocupaciones habituales (lavar pisos) se sintió bañada en sangre. Al llegar presenta una matriz de consistericia leñosa y muy dolorosa - escasa hemorragia genital. T. V. a las 21 horas: vulva ${ }_{i,}$ vagina y periné. normales; cuello largo: grueso, central. permeable a un dedo; presentación alta móvil sobre el estrecho superior. Se ordena un centigramo de morfina A las 21 más 15, el Jefe de Clínica mide una altura uterina de 38 centí. metros; la matriz tiene una consistencia leñosa y duele a permanencia. Muy poca hemorragia genital. Diag * nosticado un desprendimiénto prematura de placenta normalmente insertada se-decide intervenir por via alta. Se hacen los primeros tiempos como dis ordinario y aparece la matriz con haeras equimosis en la cara anterior: wo ineide ol segmento y se extrae un toto masculino, muerto de 2.680 gra placenta y muchos coagulos muy grandes. Se examina luégo el cuerpo uterino y se ven zonas equimóticas en la cara posterior, pero como el útero reacciona con el pitocin intramural $y$ se retrae bien, se opla por continuar ta cesárea conservando la matriz. El resto de la operación se hizo sin dificultad. Durante la intervención se inyectaron 500 c. c. de sangre. Anestesia general con ciclopropano-oxigeno. Post-operatorio con solución dextrosada, vitamina $C$. hierso y antibioticos. Salio del hospital a los-12 días en magnítieas condiciones.

\section{Observación número 3}

Servicio del Profesor Mojica. Marzo 13 de 1952.

A. L. N. de 42 años. Decimolercer embarazo. Amenorrea desde el $1^{\circ}$ de julio de 1951. Con edemas. Tensión arterial de 10 por 5. Altura uterina de -38 centímetros y de consistencia dura. Presentación cefálica alta. Ausculta. ción fetal negativa. Serología negativa. Dice el interno: *Llega el Servicio por haber presentado un dolor fuerte en el bajo vientre $y$ hemorragia genital de alguna intensidad. Al palpar el abdomen se encuentra la matriz hipertónica, dura, que impide hacer palpa ción del teto, por to que no se aprecian la presentación $y$ la posición. Ruídos fetales negativos. Se aplica un centigramo de morfina. Anotación del fefe de Clínica: sla paciente a las - seis de la tarde sintió dolor abdominal después de un vértigo y a contiruación comenzó a sangrar. Sangre toja, viva,-rutilante. Viene al hospltal con matriz endurecida, dolorosa a la palpación y con 34 centímetros de 
tiva. Examinada por el Jefe de Clínica una hora después del ingreso al hospital, la altura uterina es ya de 40 centimetros y la hemorragia continúa. Diagnosticado un désprendimiento placen'ario, de acuerdo con el Profesor, se decide operar en seguida.

Exteriorizaua la matriz, se encontraron en ella zonas equimoticas $y$ al cortar el segmento salió muchísima sangre con coágulos. Se rompieron las membranas y se extrajo un feto muerto masculino, de 2.800 gramos. Inmediatamente salió la placenta con un enorme coágulo organizado en la cara materna. Se inyectó pitocín intramuscularmente y la matriz se retrajo bien. Como no hay infiltración total y la retracción $y$ contracción son normales. no se ve la conveniencia ni la neces:dad de la histerectomía y se continúa la cesárea, dejando finalmente en la cavidad peritoneal un gramo de estreptomicina y medio millón de unidades de penicilina. Durante el acto quirúrgico se aplicaron 500 c. c. de dextrosa en agua destilada y 500 c. c. de sangre.

Anestesia general con ciclopropano y oxígeno. En el post-operatorio dextroxa en sol. salina, antibióticos, vitamina C. A los 11 días sale del hospital en completo restablecimiento.

\section{Observactón número 4}

Servicio del Protesor Acosta. Febreró 23 de 1952.

M. S. de 17 años. Primigestante de ocho meses. U. R. el 5 de junio de 1951 . Sin edemas. Tensión arterial de 10 por 6. Serología negativa. Altura uterina de 28 centímetros. Auscultcición normales; cuello parcialmente borrado, cerrado, duro. A las 10 horas ruidos cardíacos fetales múy bradicárdicos. Llega al Servicio en trabajo principiado una hora antes y dice que inmediatamente antes de venirse parc el hospital sintió una coniraccion muy larga y colorosa y que luégo sa suspendieron los intervalos de descanso de los dolores quedando un insoporta. ble dolor permanente. El útero es du. ro, de consistencia leñosa, muy sens:ble. La retracción impide la buena apreciación de las partes fetales. La auscultación letal es positiva, pero el ritmo es de 90 por minuto. Hay hemorragia vaginal moderada. Con el diagnóstico de desprendimiento prematuro de la placenta normalmente insertadc se procede inmediatamente $a$ practicar una operación cesárea.

Anestesia general con ciclo-02-eter. Disociación arciforme del segmento y extracción de un feto masculino. de 2.000 gramos, que respira espontánecmente. En la ezona inierior. (sic) de la placenta se observán grandes coágulos que ocupan una tercera parte de superificie placentaria y que confirman el desprendimiento prematuro. Se exterioriza la totalidad del útero y se oncuentra que especialmente en la zona de implantación de las trompas hay infiltración hemorrágica del músculo uterino, pero en forma moderada por lo cual se decide conservar el útero. So thtroutuce tueromente el cierno uterino dentro de la cavidad abdominal y se cierra la incisión del segmento inferior en dos planos de calquit cromado, el primero con puntos separados $y$ el segundo en sutura continua. Peritonización. Apendicectomía profiláctica. El niño murio a las cuatro ho- 
operatorio fue normal. Sale del Servicio a los-11 días por completo restablecimiento.

Las tres historias anteriores son la más clara demostración de la inutilidad inconveniencia de la histerectömia sistemática En esas pacientes con buen criterio clinico-quirurgico n? so considero necesaria ni oportuna la amputación uterind, pues la matriz no habia suirido alteraciones profundas y su dinámica era normal. ¿Por qué ser siśtemático en una conducta si en medicina no hay absolutismos y siempre hay que medir y pesar y mirar las circunstancias para resolver los casos sin sujeción incondicional a cartabones esquemáticos?

\section{Observación número 5}

Servicio del Profesor Mojica. Junio 2 de 1952.

E. Q. de 38 años. Décimo émbarazo. No da la fecha de la última regia. Hay edemas ligeros. Pulso, 140 por minuto. Tensión arterial: cero. piel pálida. Hernia umbilical. Altura uterina de 35 centimotros. Consistencia y tonicidad blandas. Presentación de vertice dere cha novil. Auscultación fetal negativis Serología negativa. Cuello corto, blando de multípara. El interno anota: - La enterma a las 5 a. m. de hoy sinthó un trastorno con angustia epigásfries. Llega al hospital $\propto$ las $2 y$ cuarto p. m. en estado de shock, con tensirn arterial de cero, pulso débil con ritmo de 140 por minuto. Hay hipotermia, gran estado anémico, desasosiego general, pero no hay dolor a ta paipactón abdominal aunque la paciente se queja de un dolor vago y es- ves de la hernia umbilical se ve ol peritoneo azul. Ligero vómito de color oscuro. Se diagnostica hemorragia interna abdominal y se prescribe sangre, graplasmoid, coramina, cortate y metrazol. Alarmado el interno por el cuadro tan grave y pensando en una hemorragia de la cavidad peritoneai de origen desconocido, llamo al Jef de Clínica. A la media hora éste en cuentra a la paciente en estado de anemia aguda, con pulso imperceptible, pero la tensión arterial ya está en 7.5 por 6 . Los ruidos cardíacos son muy velados. Los reflejos pupilares son normales. La matriz tiene ya 38 centimetros y está hipertónica, pero indolora a la palpación. y permite apreciar una presentación de vértice derecha y móvil. Al tacto vaginal: cuello de multipara que da paso a un dedo: no se puede saber si las membranas están rotas o no: presentación de vértice por encima del estrecho superior; ligera hemorragia genital, de reciente iniciación pues el Interno no la encontró en su examen. La enferma está presa-de gran-agitación y se quejá de dolor lumbar continuo. La auscultación fetal es negativa. La hipertonía uterina su crecimiento, la metrorragia y el mal estado general hacen diagnosticar desprendimiento prematuro de la placenta normalmente insertada. Mientras se prepara la intervención se continúa la transiusión y la oplicación de graplasmold, $y$ se vigila muy de cerca a la enferma. Se aprovecha el intervalo de espera para ordenar exámenes de sangre $y$ de orina, que dan el resultado siguiente:

Otina: densidad 1010, reacción ligeramente alcailina, huellas de albúmina ausencia de glucosa. Sedimento: 
gramulies $t$, leuccus $t$ células epiteliales $t+$.

Sangre: hematies 2.510.000, leucocitos 14.200 , hemoglobina $44 \%$, valor globular 0,9 . Polinucleares neutrótilos $71 \%$, eosinótilos $3 \%$, linfocitos peque ños $-15 \%$, formas de paso $11 \%$.

A las $4 \mathrm{p}$. m. la matriz tiene $40 \mathrm{cen}$ timetros de altura y su tono se ha elevado tánto que ya es imposible la palpación del teto; sigue la hemorragra genital: se visualiza más la coloración azul de la hernia umbilical, y se atribuye a invasión sanguinea a la cavidad peritoneal por existir una forma grave de útero ae Couvelaire. Después de transfundidos $1.000 \mathrm{cc}$. de sangre y 400 de graplasmoid se inicia la laparotomía: incisión vertical de la piel desde el ombligo hasta el pubis, seguida de corte de aponeurosis y disociación de los rectos anteriores. Aparece azul el peritoneo parietal, y al cortarlo se ve que hay gran cantilad de sangre en la cavidad y que a matriz está equimótica en su total:dad. Se confirma así el diagnóstico de desprendimiento prematuro de placenta. Se hace una cesárea corporal y al cortar verticalmente la matriz se inunda el campo operatorio por la abundantisima cantidad de sangre contenida en la cavidad uterina. Se e:traen feto y anexos en bloque e irturnpen coáqulos de gran tamaño. Se termina con histerectomía subtotal y con ooforectomía pues los ovarios también se hallan completamente equimóticos. E] peso del teto tue de 1.980 gramas. Anestesia con ciclopropano-oxígeno. Se continúa el tratamiento con soluciones dextrosadas y antibióticos, vitamlna $\mathrm{C}$ a la dosis de medio gramo al día, y a los doce clías la pacienie sale del hospital en buenas condicto- examen anatomopatologico.

Laboratorio Santiago Samper. Sección de Anatomía Patológica. - No 12491.

Nombre: E. Q. Servicio del docter Moilica.

Tejido, órgano o regron: placenta.

Diagnóstico elínico: apopletía úteroplacentaria.

Descripción macroscópica: la pieza se compone de una placenta que pesa 333 gramos y mide $-19 \times 18 \times 8 \times 2.2$ ctms. con un cordón umbilical de inserción paracentral que mide 38 ctms. de longitud. Tanto la placenta como el cordón umbilical son de aspecto normat.

Diagnóstico microscópico: congestión. Hemorragia. El material enviado para examen muestra placenta, completamente desarrollada. Los vasos sanguíneos están muy dilatados $\mathrm{y}$ dentro de las vellosidacies son-frecuentes los focos de hemorragia intersticial.

El Jefe de la Sección.

(Fdo.) M. Sánchez Herrerc.

Bogotá, junio 16 de 1952

\section{No 12493.}

Tejido, órgano y reglón: utero $y$ anexos.

Diagnóstico elínico: apoplojía ûteroplacentaric.

Descripcion macroscópjca: útero thisterectomía total con salpincjo-ootarectomia bilaterall que pesa 1.139 gta- 


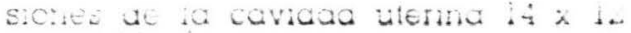

ctms. Espesor del miometrio 3,5 ctms., endometrio no hay. El cuello ha desaparecido por incorporación al segmento y sú orificio exterior tiene un diámetro de 4.2 ctms. El miometrio es de consistencia blanda, áspecto congestivo y se ven abundantes zonas de hemorragia subserosa. La cavidad endometrial contiene abundante cantidaci de coágulos sanguíneos. Los ovarios pescm cada uno 6 grms. y miden $4 \times 2$ $x 0.6$ ctms y $3.2 \times 1.8 \times 0.8$ ctms. $\mathrm{Al}$ carte en uno de ellos se ve la formación de un gran cuerpo amarillo que mide I $\times 0,6$ ctms. Las trompas miden $11 \times 0.8$ y $10 \times 0,7$ ctms. Son de aspecto normal.

Diagnóstico microscópico: embarazo. Hemoriagia uterina. El material enviado para el examen muestra útero, tubos y ovarios. En la capa interna del cuerpo del útero se observan celulas deciduales y formas de estructura que dan la impresión de-ser vellosidades placentarias. El endometrio y el miometrio, pero mucho más este- último, presentan focos, zonas o áreas de hemorragia intersticial. Las fibras muscuiares están enormemente hipertróticas. Hay edema generalizado y en ocasiones se observa ligera infiltracion leucocitaria poli y mononuclear. Los tubos y ovarios-no muestran nada especiat.

\section{(Fđo.) M. Sánchez Herrera}

Bogotá, junto 16 de 1952.

\section{Observación número 6}

\footnotetext{
Servicio del Profesor Acostá. Ábril 17 di 1952 .
}

ieminc. Uhumas regias el 20 a jul:o de 1951. Tensión arterial de 16 por 1 !. Sin edemas. Utero con tonicidad y consistencia normales y 32 ctms. de altura. Pelvimetria: 24-28-30-19. Serolo= gia negativa. Auscultación felal positiva. Iniciación del trabaio a las 23 del 16 de abril. y a las 4 más 15 a. m. del 17, dilatación cervical de un centmetro, membranas integras trabajo normal. A las 8 horas del 19 el examen obstétrico enseña una dilatación de un centímetro, vagina llena de coágulos pero no se tocan cotiledones placentarios, presentación alta. Ruidos cardíacos fetales acelerados. Se ordena reposo absoluto y aplicar dextrosa al $5 \%$ en solución salina. A las 10 y 15 la hemorragia es intensa, la lonicidad uterina normal, las contraccicnes son espaciadas, el cuello se mantiene en una dilatación de un centimetro y los ruidos cardiacos fetales so. irregulares. Se cree en la presencia de una placenta previa lateral y se hace ruptura artificial de las riembranas: la hemorragia se aminora durante unos minútos pero se réanuda con más vigor, especialmente durante Ja contracción uterina. Se descartó sil diagnóstico inicial por este sincronis. mo de hemorragia-contracción con bolsa rota y se decidió evacuar la matriz mediante una-cesárea, considerando también la irregularidad del corazón fetal. Al extraer la placenta so encontraron en su cara materna coágulos en casi toda su periferia; la matriz reaccionó al pitocín intramural. El feto salió vivo. Post-operatorio normal. Salida a los diez días.

No es aventurado pensar gue fus este un caso de desprendimiento leve. y que la hemorragia genital debiós 
al desprendimiento periférico del órgano placentario. Que no se trataba de uria placenta inferior lateral lo demostró la cesárea, en la que no se enontró la placenta adherida al segmento sino al fondo uterino. Aunqu - se intervino con la certeza diagnósica sino mas bien impelidos por la henorragia creciente y por el sufrimien- fetal la rápida conducta llevada a - evacuar la matriz con tada prontitud vino a culminar con la obtención de un feta vivo y con la profilaxia de la infiltración sanguínea en el útero. Arqumento más en pro del intervencionismo, enseñanza de que es posib!e un feto vivo si se interviene con la mayor presteza posible. Además, como se ha visto en el curso de este esbozo te monografía, no debe esperarse a que la sintomatología clásica del desprendimiento placentario aparezca. pues, cuando se hace innegable por lo evidente, ya el desprendimiento es grave y la muerte dél felo es un hecho cumplido.

\section{Observación número 7}

Servicio del Prolesor Acosta. Abril 0 de 1952.

1. M. de 39 años. Duodécimo embarazo, de seis meses. Llega en estado de anemia aguda, con edemas, tensión miterial inmensurable matriz de 25 centímetrós de altura, dura, leñosa. Henorragia genital que principió a las

p. m. La gravedad es tan evidente que inmediatamente después de su inqreso se le disecan las venas del codo para hacerle transfusión y adminis: trarle plasma y soluciones dextroseclas. A las 18 y 30 se hace operación cesárea y se encuentra que la placenta está insertada en el segmento $v$ que los ligamentos anchos, especialmente el izquierdo, tienen hematomas. Feto muerto de mil gramos. Post-ope ratorio normal y salida de! hospital a los diez días.

Como se ve por el sucinto relato de la historia precedente, no se trataba del desprendimiento prematuro d's la placenta normalmente insertada sino de un desprendimiento parcial y prematuro de la placenta inferior; pero se trae a cuento porque la matriz con su dureza leñosa aparentó el cuadro, en parte, de la sintomatología de aquella complicación. Sirve para la discusión del diagnóstico diferencial y para corroborar de la rareza del hallaz. go de signos patognomónicos.

\section{Observación número 8}

Servicio del Profesor Acosta. Julio 25 de 1951.

A. P. de C. de 23-años. Ultima regla el 28 de octubre de 1950. Sin edemas. Utero de 34 centímetros de altura, hipertónico. Auscultación fetal negativa. Tensión arterial de 11 por 8 . Pelvimetría: 21-27-30-r8. A las 3 a. m. dilatación cervical de 6 centímetros y bols $\alpha$ integra. Hemorragia genital. Estado general anémico. Como el cuello es tácilmente dilatable bajo anestesia se sompleta la dilatación y se oplica un fórceps alto para una presentación de vértice. Con gran facilidad se extrae un teto de 2.900 gramos, muerto. Inmediatamente después scle la placenta. que tiene abundantes infartos y coábulos en la cara materna. Verificado al alumbramiento se hace expresión y son expulsados cóágulos restantes. Intervenida con fórceps por presumir desprendimiento prematuro placenta 
ric. el examen de la placenta confirmó la hipótesis. El puerperio lue normal.

\section{Observación número 9}

Servicio del Protesor Acosta. No vembie 27 de 1951

E. P. de H. de 32 anos. Undecmo embarazo. Ultimas reglas el 25 de febrero. Utero blando con 36 centimetros de altura. A las 18 y 50 dilatación cervical de 4 centímetros y bolsa. integra. A las 23 se aprecia hemorragia genital de cierta consideracion y acompañada de coágulos, al tiempo que la matriz se pone dura y las contracciones se vuelven casi subintrantes. Los ruidos cardíacos fetales son normales. Se completa la dilatacion ; se aplica fórceps para una OIA ins:nuada, teniendo en cuenta también la multiparidad y la amplitud de diámetros: 24-30-32-22. Se extrae un feto con circular en el cuello y que respira espontáneamente, y como la hemorradia continúa se hace extracción manual de la placenta en breves instantes.

No especifica la historia si se examinó la placenta, talvez por la poca costumbre en nuestro medio de dejar constancia de todo lo observado y por el menosprecio que se tiene generalmente de revisar con todo cuidado los anexos: pero comparando la evolueión del trabajo con el de las otras pacientés, la súbita aparición de la hemaragria genital y la alteración del ritmo contráchll y de la dinámica uteriIna, no ulópico afirmar que hubo un desprendimiento parcial de la plocenta y que tue acertada la conducta oncominada a extraer al feto con la mayor roptdex.
E) puerperio fue normal. Madre hijo salieron del hospital a los ochs días.

\section{Observación número 10}

Servicio del Prolesor Acosta. 22 de abril de 1951.

E. F. de 40 años. Undécimo embara. zo. Ultima regla el 20 de julio de 1950. Ingresó a un servicio obstétrico independiente del Instituto de Protección Materna e Infantil, sitio en el cual tue vista por especialistas connotados en estado de suma gravedad, y en donde se le hicieron aplicaciones de urgencia. Por motivos que no interesan al aspecto obstétrico fue enviada al Instituto después de varias horas de evolución, y llega sin tensión arterial, con matriz hipertónica y dureza leñosa, 40 centimetros de altura, auscultación fetal negativa, presentación transversa y dilatación cervical casi completa. A su llegada a las 14 y 40 , y en vista de su estado precario, inhábil para una inmediata intervención, se ordena una transfusión inmediata; pero a las 16. tras un vómito sanguinolento, Ix enferma falleció.

\section{Observación número 11}

Servicio del Prolesor Acosta. Junio it de 1352 .

Para remate y final de esta casuistica dejé un caso afortumado, el último en el tiempo y quizás de los primeros por su extraordinario interes, yo que además del desprendimiento placentario otras dos complicaciones estabon presentes: procidencla del cordón y procidencia de los miembros 
superior $\epsilon$ inferior izquierdos, $y$ tras una intrincada conjunción de factores adversos, un epilogo-increíble: parto espontáneo con feto vivo. Por aquellos factorés imponiderables, ajenos a la voluntad humana la procidencia dlos miembros, que dificulta el croces. normal del encajomiento $y$ descensc de la presentación, fue lo que vino a representa la salvacion del teto. En électo, evitó la compresión del cordon entre la cabeza y el anillo del estrecho superior, $y$ así ño se interrumpleron tas comunicaciones materno fetales y siguió viva y palpitanté una esperaniza.

A. M. de N. de 24 años, con cuarto embarazo de ocho meses. Menarquia a los quince años, ciclo de 306, amenorrea desde octubre de 1951, tension: rtérial de 12 por 8 , con edemas, útero hipertónico, duro, con altura de 26 centímetros. Pelvimetría de 24-27-3020.

Llegó at Servicio a las 12 del dia con un cordón procidente, salido unos 20 centímetros fuera de la vulva, con matriz hipertónica y hemorragia genital discreta. Según relato de la enferma, el parto había comenzado a las siete de la mañana y el cordón se habia "salido" a las 10. Al tocar $e$. cordón no se percibieron pulsaciones y habia perdido su tersura $y$ brillo habituales. La auscultación fetal fue negativa. El cuello uierino esictia burido, con dilatáción de 8 centímetros, y los dedos que hacion el tacto vagina! tocaron el cordón procidente $y$ un pie del feto. Se dicanosticó una presentcción de pelvis $y$ en vista de las circunstancias anotadas se optó por defar que ol trabaio prosiguiero evolu. cionando, y para modificar el tono ute rino se ordenó aplicar dos ampolletas dé atrinal y dar un comprimico de dórico.

A las 14 más 15 las contracciones se volvieron subintrantes y la hemorragia se hizo más copiosa. Se resolvió terminar el parto haciendo una extracción podálice

Bajo anestesia general con ter $\mathrm{s}$ hiro nuevo tacto y se comprobó qu no habic presentación de pelvis sino que se trataba de una de vértice izquierda con procidencia del cordón y de los miembros superior e interior izquierdos. Se rechazaron el ple y la mano y se hizo la maniobra de Kristeller, Fácilmente se obtuvo un feto prematuro de edad aproximada de ocho meses. Se le dió por muerto y se le entregó a una enfermera mientras que se extraía la placenta con suave presión en el fondo uterino. Se examinó y se observó que la superficie de la cara materna estaba cubierta por un coágulo que ocupaba una sexta parte del área placentaria. signo de desprendimiento prematuro. Con gran sorpresa para todos los circunstantes el feto hizo un esfuerzo inspiratorio. Inmediatamente se le aspiraron mucosidades, se le practicaron maniobras de respiración attificial y se le dió oxigeno. Poco a poco el niño fue xdquiriendo un color rosado y s'd respiración se instaló normalmente. En los miembros izquierdos, superior $\theta$ interior se veían claramente cianosis $\bar{y}$ edemas.

\section{RESUMEN $\mathbf{Y}$ CONCLUSIONES}

Primera: se estudia el sindrome del desprendimiento prematuro de la placenta nornalmente insertada y se recalca sobre la -variabilidad y diversidad de la sintomatología y evolución. 
Segunda: en 10 casos se siguleron citerentes conductas:

en dos, cescrea corporal e histerec. tomia

en cuatro, cesárea segmentarta

en dos, forceps

en uno no se alcomzo a intervenit

pr whe se defo que ol parto evolu cistura espontangamente, ya que no se - zo diagnostico previo y como habia una procidencia del cordón de larga díta (dos horas) se pensó que el teto estaba-muerto.

Tercera: el desprendimiento de la placenta normalmente insertada es más frecuente en multiparas: en diez casos, siete multíparas y tres primiparas.

Cuarta: la conducta frente al desprendimienta prematuro de la placenta normalmente insertada debe ser activa y tender a la rápida evacuación uterina.

Quinta: se obtuvo feto vivo en tres casos, en uno el feto se extrajo vivo pero murio-cuatro horas despues, en los seis restantes el feto murió in útero.

Sexta: no debe ser sistemática la hisierectomia, sino subordinada al esta. do de la matriz y a su capacidad conircilit.

\section{REFERENCLAS BIBLIOGRATCAS}

(1) Amérioo Stabule: Actudizaciones de temas ginecotocológicos. $1^{a}$ serie. Póg. 74. Montevideo, 1949.

(a) Jaime Corral Maldonado: Conducis en el desprendimiento prematuro de la placenta nomalmente insertada. Rev. Col. Obst. y Gin. Vol. N $1-50 . \mathrm{Pa} \cdot 42$.

(3) Botella Llusiá: Patologia obstetricc. Pág. 448. Barcelona, 1950.

(4) Nubiola-Zárate: Tratado de Obstetricia. Págs. 721 y ss. Ed̀. Labor, 1951.

(5) S. Dexeus Font: Tratado de Obstetricia, T. II. Pág. 1919. Ed. Salvat. 1949.

(6) Manuel Luis Péres: Tratado de Obstetricia, $4^{\alpha}$ ed. Ed. López. 1943. Buenos Aires.

(7) Hernando Acosta: Conferencias de Obstetricia, 1941. Apuntes personales.

(B) Jaimo Corral Maldonado: Las grandes intervenciones obstétricas. Rev. Col. de Obst. y Gin. Vol. II. No 3. Abril-mayo 1951.

19) Rounires Morehón Rotool: Conductas médicas controvertibles Rev. Fac. de Med. de la U. N. de Bogotá. Vol. XVI. Nos. 7-8, páa. 1148.

Nota: Escrito este ensayo leí en el-No, 62 de la-Revista Espapola de Obsterrica y OINecolooia, que dirige el connotado ginecologo y experto novelista A. Ciavero Nuñez, ì segunda parte del estudio "Ginecolonia Psicosomatica" por el Dr. Francisco Haro Oareia, y en ella se dice lo siguiente: "Ya hemos - hablado anteriormente de como las emociones provoean una desviación de la masa sanguinea de la superficie cutánea al terreno del esplacaico, y cómo asi pueden determinarse roturas vasculares en el útero, especialmente por la disminución de su tono propio del embarazo. Pues bien; en ese mecanismo se basa Schwarz para admitir que una emoción pueda determinar un hematoma retroplacentario, y Salerno vuetve de nikevo sobre este punto de vista de tas reacciones circulatorias por efectos desplacientes o displicentes (la palabra displaciente, usada por lo general, es incorrecta) para justificar este aceldente, que si ocurre en los primeros mesés del embarazo originará el aborto, y si es mís adefante-el sindrome del desprendimiento prematuro de la placenta normalmente insertada, que, actualmeate, se relaciona casi en absoluto con las toxemias gravidicas", 